Article

\title{
Large Constant-Sign Solutions of Discrete Dirichlet Boundary Value Problems with $p$-Mean Curvature Operator
}

\author{
Jianxia Wang ${ }^{1,2}$ and Zhan Zhou ${ }^{1,2, * \mathbb{D}}$ \\ 1 School of Mathematics and Information Science, Guangzhou University, Guangdong 510006, China; \\ jxwang@gzhu.edu.cn \\ 2 Center for Applied Mathematics, Guangzhou University, Guangdong 510006, China \\ * Correspondence: zzhou@gzhu.edu.cn
}

Received: 3 February 2020; Accepted: 5 March 2020; Published: 9 March 2020

\begin{abstract}
In this paper, we consider the existence of infinitely many large constant-sign solutions for a discrete Dirichlet boundary value problem involving $p$-mean curvature operator. The methods are based on the critical point theory and truncation techniques. Our results are obtained by requiring appropriate oscillating behaviors of the non-linear term at infinity, without any symmetry assumptions.
\end{abstract}

Keywords: discrete Dirichlet boundary value problem; $p$-mean curvature operator; constant-sign solutions; discrete maximum principle; critical point theory

\section{Introduction}

Let $\mathbb{Z}, \mathbb{N}$ and $\mathbb{R}$ denote the sets of integer numbers, natural numbers and real numbers, respectively. For $a, b \in \mathbb{Z}$, define $\mathbb{Z}(a)=\{a, a+1, \cdots\}$, and $\mathbb{Z}(a, b)=\{a, a+1, \cdots, b\}$ when $a \leq b$.

Consider the following Dirichlet boundary value problem of the nonlinear difference equation

$$
\left(D_{p}^{\lambda, f}\right)\left\{\begin{array}{l}
-\triangle\left(\phi_{p, c}(\triangle u(k-1))\right)=\lambda f(k, u(k)), \quad k \in \mathbb{Z}(1, T), \\
u(0)=u(T+1)=0
\end{array}\right.
$$

where $T$ is a given positive integer, $\lambda$ is a positive real parameter, $\triangle$ is the forward difference operator defined by $\triangle u(k)=u(k+1)-u(k), f(k, \cdot): \mathbb{R} \rightarrow \mathbb{R}$ is a continuous function for each $k \in \mathbb{Z}(1, T)$ and $\phi_{p, c}(s):=\left(1+|s|^{2}\right)^{\frac{p-2}{2}} s, p \in[1,+\infty)$. Here, $\triangle\left(\phi_{p, c}(\triangle u(k-1))\right)$ may be seen as a discretization of the $p$-mean curvature operator.

We may think problem $\left(D_{p}^{\lambda, f}\right)$ as being a discrete analog of one-dimensional case of the following problem

$$
\begin{cases}-\operatorname{div}\left(\phi_{p, c}(\nabla u)\right)=\lambda f(x, u), & x \in \Omega \subset \mathbb{R}^{n} \\ u=0, & x \in \partial \Omega,\end{cases}
$$

where $\operatorname{div}\left(\phi_{p, c}(\nabla u)\right)$ is named $p$-mean curvature operator, which is a generalization of mean curvature operator; see [1,2]. If $p=1$, it reduces to the mean curvature operator. If $p=2$, it reduces to the Laplacian operator. The above problem arises from differential geometry and physics such as capillarity; see [3-5] and references therein. When $p=1$ and $f(x, u)=u$, the above problem describes the free surface of a pendent drop filled with liquid under gravitational field [4]. In the past decades, several authors have discussed the existence and multiplicity of solutions of Problem (1); see [1,6-12]. For example, Chen and Shen in [1] have obtained the existence of infinitely many solutions of Problem (1) with $\lambda=1$ via a symmetric version of Mountain Pass Theorem. When $p=1$ and $\Omega=(0,1)$, 
Obersnel and Omari in [11] have established the existence and multiplicity of positive solutions of Problem (1), which depend on the behavior of $f$ at zero or at infinity. G. A. Afrouzi et al. in [6] have acquired a sequence of nonnegative and nontrivial solutions strongly converging to zero in $C^{1}([0,1])$, under suitable oscillating behavior of the nonlinear term $f$ at zero. However, the results on the existence of solutions for problem $\left(D_{p}^{\lambda, f}\right)$ are scarce in the literature besides the case of $p=1$.

Nonlinear discrete problems appear in many mathematical models, such as computer science, mechanical engineering, control systems, artificial or biological neural networks, economics, fluid mechanics and many others; see [13-17]. Many authors have discussed the existence and multiplicity of solutions for difference equations through classical tools of nonlinear analysis: Fixed point theorems, upper and lower solutions techniques; see [7,9] and the references given therein. Since 2003, by starting from the seminal paper [18], variational methods have been used to investigate nonlinear difference equations, which have obtained various results; see [19-34].

In paper [35], the authors have considered problem $\left(D_{1}^{\lambda, f}\right)$, obtaining infinitely many positive solutions when $\lambda$ belongs to a precise real interval. It is worth noticing that the suitable oscillating behaviors of the nonlinear term $f$ at infinity play a key role. Inspired by [19,32,35-40], the main purpose of this paper is to investigate the existence conditions of infinitely many constant-sign solutions for problem $\left(D_{p}^{\lambda, f}\right)$, without any symmetry hypothesis. Here, a solution $\{u(k)\}$ of $\left(D_{p}^{\lambda, f}\right)$ is called a constant-sign solution, if $u(k)>0$ for all $k \in \mathbb{Z}(1, T)$ or $u(k)<0$ for all $k \in \mathbb{Z}(1, T)$. Compared to problem $\left(D_{1}^{\lambda, f}\right)$, problem $\left(D_{p}^{\lambda, f}\right)$ is more difficult to handle. To facilitate the analysis, we have to divide the problem into two categories: $1 \leq p<2$ and $2 \leq p<+\infty$. We believe that this is the first time to discuss the existence of infinitely many solutions for a non-linear second order difference equation with $p$-mean curvature operator.

A special case of our results is the following.

Theorem 1. Let $g: \mathbb{R} \rightarrow \mathbb{R}$ be a continuous function such that $g(t) t \geq 0$ for $t \neq 0$. Assume that

$$
\liminf _{t \rightarrow \infty} \frac{\int_{0}^{t} g(\tau) d \tau}{|t|^{p}}=0, \text { and } \limsup _{t \rightarrow \infty} \frac{\int_{0}^{t} g(\tau) d \tau}{|t|^{p}}=+\infty .
$$

Then, for every $\lambda>0$, the problem

$$
\left\{\begin{array}{l}
-\triangle\left(\phi_{p, c}(\triangle u(k-1))\right)=\lambda g(u(k)), \quad k \in \mathbb{Z}(1, T), \\
u(0)=u(T+1)=0,
\end{array}\right.
$$

admits two unbounded sequences of constant-sign solutions (one positive and one negative).

This paper is organized as follows. In Section 2, we introduce the the suitable Banach space and appropriate functional corresponding to problem $\left(D_{p}^{\lambda, f}\right)$. To obtain sequences of constant-sign solutions of problem $\left(D_{p}^{\lambda, f}\right)$, three basic lemmas are introduced. In Section 3 , under suitable hypotheses on $f$, we obtain the existence of infinitely many constant-sign solutions for problem $\left(D_{p}^{\lambda, f}\right)$. In Section 4 , we give two examples to demonstrate our results. Finally, conclusions are given for this paper.

\section{Mathematical Background}

To solve problem $\left(D_{p}^{\lambda, f}\right)$, we naturally select the $T$-dimensional Banach space

$$
X=\{u: \mathbb{Z}(0, T+1) \rightarrow \mathbb{R}: u(0)=u(T+1)=0\},
$$

endowed with the norm

$$
\|u\|:=\left(\sum_{k=1}^{T}(\triangle u(k))^{2}\right)^{\frac{1}{2}} \text { for all } u \in X
$$


Another useful norm on $\mathrm{X}$ is

$$
\|u\|_{\infty}:=\max _{k \in \mathbb{Z}(1, T)}|u(k)| \text { for all } u \in X
$$

In the sequel, we will use the following inequalities.

For $0<r<s, x_{k} \geq 0, k \in \mathbb{Z}(1, n)$, one has

$$
\left(\sum_{k=1}^{n} x_{k}^{s}\right)^{1 / s} \leq\left(\sum_{k=1}^{n} x_{k}^{r}\right)^{1 / r}
$$

see [41].

$$
\|u\|_{\infty} \leq \frac{\sqrt{T+1}}{2}\|u\|
$$

for every $u \in X$, it can follow from Lemma 2.2 of [42].

For all $u \in X$, let

$$
\Phi(u):=\frac{1}{p} \sum_{k=0}^{T}\left(\left(1+(\triangle u(k))^{2}\right)^{\frac{p}{2}}-1\right), \text { and } \Psi(u):=\sum_{k=1}^{T} F(k, u(k)),
$$

where $F(k, t):=\int_{0}^{t} f(k, \tau) d \tau$ for every $t \in \mathbb{R}$ and $k \in \mathbb{Z}(1, T)$. Further, let us denote $I_{\lambda}(u):=\Phi(u)-\lambda \Psi(u)$ for $u \in X$. Through standard arguments, we follow that $I_{\lambda} \in C^{1}(S, \mathbb{R})$, and the critical points of $I_{\lambda}$ are exactly the solutions of problem $\left(D_{p}^{\lambda, f}\right)$. In fact, one has

$$
\begin{aligned}
I_{\lambda}^{\prime}(u)(v) & =\sum_{k=0}^{T}\left(\phi_{p, c}(\triangle u(k)) \triangle v(k)-\lambda \sum_{k=1}^{T} f(k, u(k)) v(k)\right. \\
& =\sum_{k=0}^{T}\left(\phi_{p, c}(\triangle u(k)) v(k+1)-\sum_{k=0}^{T}\left(\phi_{p, c}(\triangle u(k)) v(k)-\lambda \sum_{k=1}^{T} f(k, u(k)) v(k)\right.\right. \\
& =\sum_{k=1}^{T}\left(\phi_{p, c}(\triangle u(k-1)) v(k)-\sum_{k=1}^{T}\left(\phi_{p, c}(\triangle u(k)) v(k)-\lambda \sum_{k=1}^{T} f(k, u(k)) v(k)\right.\right. \\
& =-\sum_{k=1}^{T}\left[\triangle \left(\left(\phi_{p, c}(\triangle u(k))-\lambda f(k, u(k))\right] v(k),\right.\right.
\end{aligned}
$$

for all $u, v \in X$.

Next, we need to establish the following strong maximum principle to obtain the positive solutions of problem $\left(D_{p}^{\lambda, f}\right)$, i.e., $u(k)>0$ for each $k \in \mathbb{Z}(1, T)$.

Lemma 1. Assume $u \in X$ such that either

$$
u(k)>0 \text { or }-\triangle\left(\varphi_{p, c}(\triangle u(k-1)) \geq 0\right.
$$

for any $k \in \mathbb{Z}(1, T)$. Then, either $u>0$ in $\mathbb{Z}(1, T)$ or $u \equiv 0$.

Proof. For $u \in X$, put $m=\min \{u(k), k \in \mathbb{Z}(0, T+1)\}$, then $m \leq 0$.

If there exists $j \in \mathbb{Z}(1, T)$ such that $u(j)=m$, we claim that $u \equiv 0$. Indeed, since $\Delta u(j-1)=$ $u(j)-u(j-1) \leq 0$ and $\Delta u(j)=u(j+1)-u(j) \geq 0, \varphi_{p, c}(s)$ is strictly monotone increasing in $s$, and $\varphi_{p, c}(0)=0$, we have

$$
\varphi_{p, c}(\triangle u(j)) \geq 0 \geq \varphi_{p, c}(\triangle u(j-1))
$$


On the other hand, by (5), let $k=j$, we obtain

$$
\varphi_{p, c}(\triangle u(j)) \leq \varphi_{p, c}(\triangle u(j-1)) .
$$

Combining inequalities (6) and (7), we get that $\varphi_{p, c}(\triangle u(j))=0=\varphi_{p, c}(\Delta u(j-1))$. That is $u(j+1)=u(j-1)=u(j)=m$. By iterating this argument, we obtain easily $u(0)=u(1)=u(2)=$ $\ldots=u(T)=u(T+1)$. Thus $u \equiv 0$.

If $u(j)>m$ for every $j \in \mathbb{Z}(1, T)$, then $u(0)=u(T+1)=m=0$. It follows that $u(j)>0$, for all $j \in \mathbb{Z}(1, T)$. The proof is complete.

In the same way, we have the following result to get negative solutions problem $\left(D_{p}^{\lambda, f}\right)$, i.e., $u(k)<$ 0 for each $k \in \mathbb{Z}(1, T)$.

Lemma 2. Assume $u \in X$ such that either

$$
u(k)<0 \text { or }-\triangle\left(\varphi_{p, c}(\triangle u(k-1)) \leq 0,\right.
$$

for any $k \in \mathbb{Z}(1, T)$. Then, either $u<0$ in $\mathbb{Z}(1, T)$ or $u \equiv 0$.

Truncation techniques are usually used to discuss the existence of constant-sign solutions. To the end, we introduce the following truncations of the functions $f(k, t)$ for every $k \in \mathbb{Z}(1, T)$. If $f(k, 0) \geq 0$ for each $k \in \mathbb{Z}(1, T)$. Set

$$
f^{+}(k, t):= \begin{cases}f(k, t), & \text { if } t \geq 0, \\ f(k, 0), & \text { if } t<0 .\end{cases}
$$

Clearly, $f^{+}(k, \cdot)$ is also continuous, for every $k \in \mathbb{Z}(1, T)$. By Lemma 1 , all solutions of problem $\left(D_{p}^{\lambda, f^{+}}\right)$are also solutions of problem $\left(D_{p}^{\lambda, f}\right)$. Therefore, when problem $\left(D_{p}^{\lambda, f^{+}}\right)$has non-zero solutions, then problem $\left(D_{p}^{\lambda, f}\right)$ possesses positive solutions.

If $f(k, 0) \leq 0$ for each $k \in \mathbb{Z}(1, T)$. Set

$$
f^{-}(k, t):= \begin{cases}f(k, 0), & \text { if } t>0 \\ f(k, t), & \text { if } t \leq 0 .\end{cases}
$$

When problem $\left(D_{p}^{\lambda, f^{-}}\right)$has non-zero solutions, then problem $\left(D_{p}^{\lambda, f}\right)$ possesses negative solutions. $\left(D_{p}^{\lambda, f}\right)$.

Here, we introduce a lemma (Theorem 4.3 of [38]) which is the main tool used to research problem

Lemma 3. Let $X$ be a finite dimensional Banach space and let $I_{\lambda}: X \rightarrow \mathbb{R}$ be a function satisfying the following structure hypothesis:

$(\mathrm{H}) I_{\lambda}(u):=\Phi(u)-\lambda \Psi(u)$ for all $u \in X$, where $\Phi, \Psi: X \rightarrow \mathbb{R}$ be two continuously Gâteux differentiable functions with $\Phi$ coercive, i.e., $\lim _{\|u\| \rightarrow+\infty} \Phi(u)=+\infty$, and such that $\inf _{X} \Phi=\Phi(0)=$ $\Psi(0)=0$.

For all $r>0$, put

$$
\varphi(r):=\frac{\sup _{\Phi^{-1}[0, r]} \Psi}{r} \text {, and } \varphi_{\infty}:=\liminf _{r \rightarrow+\infty} \varphi(r) .
$$

Assume that $\varphi_{\infty}<+\infty$ and for each $\lambda \in\left(0, \frac{1}{\varphi_{\infty}}\right) I_{\lambda}$ is unbounded from below. Then, there is a sequence $\left\{u_{n}\right\}$ of critical points (local minima) of $I_{\lambda}$ such that $\lim _{n \rightarrow+\infty} \Phi\left(u_{n}\right)=+\infty$. 


\section{Main Results}

In the following, we will discuss the existence of constant-sign solutions of problem $\left(D_{p}^{\lambda, f}\right)$. Our purpose is to apply Lemma 3 to the function $I_{\lambda}^{ \pm}: X \rightarrow \mathbb{R}, I_{\lambda}^{ \pm}(u):=\Phi(u)-\lambda \Psi \pm(u)$, where $\Psi^{ \pm}(u)=\sum_{k=1}^{T} F^{ \pm}(k, u(k))$ and $F^{ \pm}(k, t):=\int_{0}^{t} f^{ \pm}(k, \tau) d \tau$ for every $k \in \mathbb{Z}(1, T)$ and then exploit Lemma 1 or Lemma 2 to get our results.

Let

$$
A_{ \pm \infty}:=\liminf _{t \rightarrow+\infty} \frac{\sum_{k=1}^{T} \max _{0 \leq s \leq t} F(k, \pm s)}{t^{p}} \text {, and } B^{ \pm \infty}:=\limsup _{t \rightarrow \pm \infty} \frac{\sum_{k=1}^{T} F(k, t)}{|t|^{p}} .
$$

Considering the functional $I_{\lambda}^{+}$, we have the following conclusions.

Theorem 2. Let $1 \leq p<2$ and $f(k, \cdot): \mathbb{R} \rightarrow \mathbb{R}$ to be a continuous function with $f(k, 0) \geq 0$ for each $k \in \mathbb{Z}(1, T)$. Assume that

$$
\left(i_{1}\right) A_{+\infty}<\frac{2^{p-1}}{(T+1)^{\frac{p}{2}}} B^{+\infty}
$$

Then, for each $\lambda \in\left(\frac{2}{p B^{+\infty}}, \frac{2^{p}}{p(T+1)^{\frac{p}{2}} A_{+\infty}}\right)$, problem $\left(D_{p}^{\lambda, f}\right)$ has an unbounded sequence of positive solutions.

Proof. Consider the auxiliary problem

$$
\left(D_{p}^{\lambda, f^{+}}\right)\left\{\begin{array}{l}
-\triangle\left(\phi_{p, c}(\triangle u(k-1))\right)=\lambda f^{+}(k, u(k)), \quad k \in \mathbb{Z}(1, T), \\
u(0)=u(T+1)=0
\end{array}\right.
$$

Obviously $\Phi$ and $\Psi^{+}$satisfy hypothesis required in Lemma 3. For $t>0$, set

$$
r=\frac{1}{p}\left(\sqrt{\frac{4 t^{2}}{T+1}+(T+1)^{\frac{2 p-2}{p}}}-(T+1)^{\frac{p-1}{p}}\right)^{p}
$$

Assume $u \in X$ and

$$
\Phi(u)=\frac{1}{p} \sum_{k=0}^{T}\left(\left(1+(\triangle u(k))^{2}\right)^{\frac{p}{2}}-1\right) \leq r .
$$

Put $v(k)=\left(1+(\triangle u(k))^{2}\right)^{\frac{p}{2}}-1$, for every $k \in \mathbb{Z}(0, T)$, then $\sum_{k=0}^{T} v(k) \leq p r$.

By (2) and Hölder inequality as well, we have

$$
\begin{aligned}
\sum_{k=0}^{T}(\Delta u(k))^{2} & =\sum_{k=0}^{T}\left(\left((1+v(k))^{\frac{1}{p}}\right)^{2}-1\right) \\
& \leq\left(\sum_{k=0}^{T} v(k)\right)^{\frac{2}{p}}+2(T+1)^{\frac{p-1}{p}}\left(\sum_{k=0}^{T} v(k)\right)^{\frac{1}{p}} \\
& \leq(p r)^{\frac{2}{p}}+2(T+1)^{\frac{p-1}{p}}(p r)^{\frac{1}{p}} \\
& =\frac{4 t^{2}}{T+1} .
\end{aligned}
$$

Owing to (3), it follows

$$
\|u\|_{\infty} \leq \frac{\sqrt{T+1}}{2}\left(\sum_{k=0}^{T}(\triangle u(k))^{2}\right)^{\frac{1}{2}} \leq t
$$


Thus, one has $\Phi^{-1}[0, r] \subseteq\left\{u \in X:\|u\|_{\infty} \leq t\right\}$

By the definition of $\varphi$, we obtain

$$
\varphi(r)=\frac{\sup _{\Phi^{-1}[0, r]} \Psi^{+}}{r} \leq \frac{\sup _{\|u\|_{\infty} \leq t} \sum_{k=0}^{T} F^{+}(k, u(k))}{r} \leq \frac{p \sum_{k=1}^{T} \max _{0 \leq s \leq t} F(k, s)}{\left(\sqrt{\frac{4 t^{2}}{T+1}+(T+1)^{\frac{2 p-2}{p}}}-(T+1)^{\frac{p-1}{p}}\right)^{p}} .
$$

Bearing in mind condition $\left(i_{1}\right)$, we follow that $\varphi_{\infty} \leq \frac{p(T+1)^{\frac{p}{2}}}{2^{p}} A_{+\infty}<+\infty$.

In the next step, we need to prove that $I_{\lambda}^{+}$is unbounded from below. To this end, we consider two cases: $B^{+\infty}=+\infty$ and $B^{+\infty}<+\infty$. If $B^{+\infty}=+\infty$, let $\left\{c_{n}\right\}$ be a sequence of positive numbers, with $\lim _{n \rightarrow+\infty} c_{n}=+\infty$, such that

$$
\sum_{k=1}^{T} F^{+}\left(k, c_{n}\right)=\sum_{k=1}^{T} F\left(k, c_{n}\right) \geq \frac{(2+p)}{\lambda p} c_{n}^{p}, \text { for every } n \in \mathbb{N}
$$

In the following, we take in $X$ the sequence $\left\{\omega_{n}\right\}$ defined by putting $\omega_{n}(k)=c_{n}$, for $k \in \mathbb{Z}(1, T)$. Using again (2), one has

$$
I_{\lambda}^{+}\left(\omega_{n}\right)=\frac{2}{p}\left(\left(1+c_{n}^{2}\right)^{\frac{p}{2}}-1\right)-\lambda \sum_{k=1}^{T} F^{+}\left(k, c_{n}\right) \leq \frac{2}{p} c_{n}^{p}-\frac{2+p}{p} c_{n}^{p}=-c_{n}^{p}
$$

which implies that $\lim _{n \rightarrow+\infty} I_{\lambda}^{+}\left(\omega_{n}\right)=-\infty$. If $B^{+\infty}<+\infty$, since $\lambda>\frac{2}{p B^{+\infty}}$, we may take $\epsilon_{0}>0$ such that $\frac{2}{p}-\lambda B^{+\infty}+\lambda \epsilon_{0}<0$. Then there exists a sequence of positive numbers $\left\{c_{n}\right\}$ such that $\lim _{n \rightarrow+\infty} c_{n}=+\infty$ and

$$
\left(B^{+\infty}-\epsilon_{0}\right) c_{n}^{p} \leq \sum_{k=1}^{T} F^{+}\left(k, c_{n}\right)=\sum_{k=1}^{T} F\left(k, c_{n}\right) \leq\left(B^{+\infty}+\epsilon_{0}\right) c_{n}^{p}
$$

Arguing as before and by choosing $\left\{\omega_{n}\right\}$ in $X$ as above, we have

$$
I_{\lambda}^{+}\left(\omega_{n}\right)=\frac{2}{p}\left(\left(1+c_{n}^{2}\right)^{\frac{p}{2}}-1\right)-\lambda \sum_{k=1}^{T} F^{+}\left(k, c_{n}\right) \leq \frac{2}{p} c_{n}^{p}-\lambda\left(B^{+\infty}-\epsilon_{0}\right) c_{n}^{p}=\left(\frac{2}{p}-\lambda B^{+\infty}+\lambda \epsilon_{0}\right) c_{n}^{p} .
$$

Since $\frac{2}{p}-\lambda B^{+\infty}+\lambda \epsilon_{0}<0$, it is clear that $\lim _{n \rightarrow+\infty} I_{\lambda}^{+}\left(\omega_{n}\right)=-\infty$. Considering the above two cases, we follow that $I_{\lambda}^{+}$is unbounded from below.

According to Lemma 3, there exist a sequence $\left\{u_{n}\right\}$ of critical points (local minima) of $I_{\lambda}^{+}$such that $\lim _{n \rightarrow+\infty} \Phi\left(u_{n}\right)=+\infty$. Hence, for every $n \in \mathbb{N}, u_{n}$ is a non-zero solution of problem $\left(D_{p}^{\lambda, f^{+}}\right)$, by Lemma $1, u_{n}$ is a positive solution of problem $\left(D_{p}^{\lambda, f}\right)$. Since $\Phi$ is bounded on bounded sets and $\lim _{n \rightarrow+\infty} \Phi\left(u_{n}\right)=+\infty,\left\{u_{n}\right\}$ must be unbounded. So Theorem 2 holds and the proof is complete.

Theorem 3. Let $2 \leq p<+\infty$ and $f(k, \cdot): \mathbb{R} \rightarrow \mathbb{R}$ to be a continuous function with $f(k, 0) \geq 0$ for each $k \in \mathbb{Z}(1, T)$. Assume that

$$
\left(i_{2}\right) A_{+\infty}<\frac{(\sqrt{2})^{p}}{(T+1)^{p-1}} B^{+\infty}
$$
solutions.

Then, for each $\lambda \in\left(\frac{(\sqrt{2})^{p}}{p B^{+\infty}}, \frac{2^{p}}{p(T+1)^{p-1} A_{+\infty}}\right)$, problem $\left(D_{p}^{\lambda, f}\right)$ has an unbounded sequence of positive 
Proof. We sketch only the differences with the proof of Theorem 2 . For $t>0$, make

$$
r=\frac{(2 t)^{p}}{p(T+1)^{p-1}}
$$

Assume $u \in X$ and

$$
\Phi(u)=\frac{1}{p} \sum_{k=0}^{T}\left(\left(1+(\triangle u(k))^{2}\right)^{\frac{p}{2}}-1\right) \leq r .
$$

Denote $v(k)=\left(1+(\triangle u(k))^{2}\right)^{\frac{p}{2}}-1$, for every $k \in \mathbb{Z}(0, T)$, then $\sum_{k=0}^{T} v(k) \leq p r$.

Noting the inequality $(x+y)^{\theta} \leq x^{\theta}+y^{\theta}$, for $0<\theta \leq 1, x \geq 0, y \geq 0$ and Hölder inequality, one has

$$
\begin{aligned}
\sum_{k=0}^{T}(\triangle u(k))^{2} & \left.=\sum_{k=0}^{T}(1+v(k))^{\frac{2}{p}}-1\right) \\
& \leq \sum_{k=0}^{T}(v(k))^{\frac{2}{p}} \\
& \leq(T+1)^{\frac{p-2}{p}}\left(\sum_{k=0}^{T} v(k)\right)^{\frac{2}{p}} \\
& \leq(T+1)^{\frac{p-2}{p}}(p r)^{\frac{2}{p}}=\frac{4 t^{2}}{T+1}
\end{aligned}
$$

Applying (3), we have

$$
\|u\|_{\infty} \leq \frac{\sqrt{T+1}}{2}\left(\sum_{k=0}^{T}\left(\Delta u_{k}\right)^{2}\right)^{\frac{1}{2}} \leq t
$$

By the definition of $\varphi$, we have

$$
\varphi(r)=\frac{\sup _{\Phi^{-1}[0, r]} \Psi^{+}}{r} \leq \frac{\sup _{\|u\|_{\infty} \leq t} \sum_{k=0}^{T} F^{+}(k, u(k))}{r} \leq \frac{p(T+1)^{p-1} \sum_{k=1}^{T} \max _{0 \leq s \leq t} F(k, s)}{2^{p} t^{p}} .
$$

Using condition $\left(i_{2}\right), \varphi_{\infty} \leq \frac{p(T+1)^{p-1}}{2^{p}} A_{+\infty}<+\infty$ holds.

Now, we verify that $I_{\lambda}^{+}$is unbounded form blow. Fist, assume that $B^{+\infty}=+\infty$. Let $\left\{c_{n}\right\}$ be a sequence of positive numbers, with $\lim _{n \rightarrow+\infty} c_{n}=+\infty$, such that

$$
\sum_{k=1}^{T} F^{+}\left(k, c_{n}\right)=\sum_{k=1}^{T} F\left(k, c_{n}\right) \geq \frac{(\sqrt{2})^{p}+p}{\lambda p} c_{n}^{p}, \text { for } n \in \mathbb{N}
$$

Picking the sequence $\left\{\omega_{n}\right\}$ in $X$ by $\omega_{n}(k)=c_{n}$, for $k \in \mathbb{Z}(1, T)$. Exploiting the inequality $(x+y)^{\theta} \leq 2^{\theta-1}\left(x^{\theta}+y^{\theta}\right)$ for $\theta \geq 1, x \geq 0, y \geq 0$, we get

$$
\begin{aligned}
I_{\lambda}^{+}\left(\omega_{n}\right) & =\frac{2}{p}\left(\left(1+c_{n}^{2}\right)^{\frac{p}{2}}-1\right)-\lambda \sum_{k=1}^{T} F^{+}\left(k, c_{n}\right) \leq \frac{(\sqrt{2})^{p}}{p} c_{n}^{p}+\frac{(\sqrt{2})^{p}-2}{p}-\frac{(\sqrt{2})^{p}+p}{p} c_{n}^{p} \\
& =-c_{n}^{p}+\frac{(\sqrt{2})^{p}-2}{p},
\end{aligned}
$$

which implies that $\lim _{n \rightarrow+\infty} I_{\lambda}\left(\omega_{n}\right)=-\infty$. 
Next, assume that $B^{+\infty}<+\infty$. Since $\lambda>\frac{(\sqrt{2})^{p}}{p B^{+\infty}}$, we may take $\epsilon_{0}>0$ such that $\frac{(\sqrt{2})^{p}}{p}-\lambda B^{\infty}+$ $\lambda \epsilon_{0}<0$. Then there exists a sequence of positive numbers $\left\{c_{n}\right\}$ such that $\lim _{n \rightarrow+\infty} c_{n}=+\infty$ and

$$
\left(B^{+\infty}-\epsilon_{0}\right) c_{n}^{p} \leq \sum_{k=1}^{T} F^{+}\left(k, c_{n}\right)=\sum_{k=1}^{T} F\left(k, c_{n}\right) \leq\left(B^{+\infty}+\epsilon_{0}\right) c_{n}^{p}
$$

Define the sequence $\left\{\omega_{n}\right\}$ in $S$ as above, we obtain

$$
\begin{aligned}
I_{\lambda}^{+}\left(\omega_{n}\right) & =\frac{2}{p}\left(\left(1+c_{n}^{2}\right)^{\frac{p}{2}}-1\right)-\lambda \sum_{k=1}^{T} F^{+}\left(k, c_{n}\right) \leq \frac{(\sqrt{2})^{p}}{p} c_{n}^{p}+\frac{(\sqrt{2})^{p}-2}{p}-\lambda\left(B^{+\infty}-\epsilon_{0}\right) c_{n}^{p} \\
& =\left(\frac{(\sqrt{2})^{p}}{p}-\lambda B^{+\infty}+\lambda \epsilon_{0}\right) c_{n}^{p}+\frac{(\sqrt{2})^{p}-2}{p} .
\end{aligned}
$$

Since $\frac{(\sqrt{2})^{p}}{p}-\lambda B^{+\infty}+\lambda \epsilon_{0}<0$, it is obvious that $\lim _{n \rightarrow+\infty} I_{\lambda}^{+}\left(\omega_{n}\right)=-\infty$.

Thus, we follow that $I_{\lambda}^{+}$is unbounded from below. According to Lemmas 1 and 3, we have finished the proof of the theorem.

Similarly, considering the functional $I_{\lambda}^{-}$, we can achieve the following results.

Theorem 4. Let $1 \leq p<2$ and $f(k, \cdot): \mathbb{R} \rightarrow \mathbb{R}$ to be a continuous function with $f(k, 0) \leq 0$ for each $k \in \mathbb{Z}(1, T)$. Assume that

$$
\left(i_{3}\right) A_{-\infty}<\frac{2^{p-1}}{(T+1)^{\frac{p}{2}}} B^{-\infty}
$$

Then, for each $\lambda \in\left(\frac{2}{p B^{-\infty}}, \frac{2^{p}}{p(T+1)^{\frac{p}{2}} A_{-\infty}}\right)$, problem $\left(D_{p}^{\lambda, f}\right)$ has an unbounded sequence of negative solutions.

Theorem 5. Let $2 \leq p<+\infty$ and $f(k, \cdot): \mathbb{R} \rightarrow \mathbb{R}$ to be a continuous function with $f(k, 0) \leq 0$ for each $k \in \mathbb{Z}(1, T)$. Assume that

$$
\left(i_{4}\right) A_{-\infty}<\frac{(\sqrt{2})^{p}}{(T+1)^{p-1}} B^{-\infty}
$$
solutions.

Then, for each $\lambda \in\left(\frac{(\sqrt{2})^{p}}{p B^{-\infty}}, \frac{2^{p}}{p(T+1)^{p-1} A_{-\infty}}\right)$, problem $\left(D_{p}^{\lambda, f}\right)$ has an unbounded sequence of negative

Combining Theorems 2 and 4, we have the following corollary.

Corollary 1. Let $1 \leq p<2$ and $f(k, \cdot): \mathbb{R} \rightarrow \mathbb{R}$ to be a continuous function with $f(k, 0)=0$ for each $k \in \mathbb{Z}(1, T)$. Assume that

$$
\left(i_{5}\right) \max \left\{A_{+\infty}, A_{-\infty}\right\}<\frac{2^{p-1}}{(T+1)^{\frac{p}{2}}} \min \left\{B^{+\infty}, B^{-\infty}\right\} .
$$

Then, for each $\lambda \in\left(\frac{2}{p \min \left\{B^{+\infty}, B^{-\infty}\right\}}, \frac{2^{p}}{p(T+1)^{\frac{p}{2}} \max \left\{A_{+\infty}, A_{-\infty}\right\}}\right)$, problem $\left(D_{p}^{\lambda, f}\right)$ admits two unbounded sequences of constant-sign solutions (one positive and one negative).

Similarly, combining Theorems 3 and 5, we have the following corollary.

Corollary 2. Let $2 \leq p<+\infty$ and $f(k, \cdot): \mathbb{R} \rightarrow \mathbb{R}$ to be a continuous function with $f(k, 0)=0$ for each $k \in \mathbb{Z}(1, T)$. Assume that

$$
\left(i_{6}\right) \max \left\{A_{+\infty}, A_{-\infty}\right\}<\frac{(\sqrt{2})^{p}}{(T+1)^{p-1}} \min \left\{B^{+\infty}, B^{-\infty}\right\} .
$$


Then, for each $\lambda \in\left(\frac{(\sqrt{2})^{p}}{p \min \left\{B^{+\infty}, B^{-\infty}\right\}}, \frac{2^{p}}{p(T+1)^{p-1} \max \left\{A_{+\infty}, A_{-\infty}\right\}}\right)$, problem $\left(D_{p}^{\lambda, f}\right)$ admits admits two unbounded sequences of constant-sign solutions (one positive and one negative).

Remark 1. If we let $p \rightarrow 2^{-}$in Theorem 2, we find that the conditions and consequence of Theorem 2 is the same as those of Theorem 3 for $p=2$. Moreover the results are consistent with results in [37]. For the special case, $p=1$, Theorem 2 reduces to Corollary 2.1 of [35].

Remark 2. We note that, if for each $k \in \mathbb{Z}(1, T), f(k, \cdot): \mathbb{R} \rightarrow \mathbb{R}$ is a continuous function satisfying $f(k, t) t \geq 0$ for all $t \in \mathbb{R} \backslash\{0\}$, then

$$
A_{+\infty}=\liminf _{t \rightarrow+\infty} \frac{\sum_{k=1}^{T} F(k, t)}{t^{p}} \text {, and } A_{-\infty}=\liminf _{t \rightarrow-\infty} \frac{\sum_{k=1}^{T} F(k, t)}{|t|^{p}} .
$$

Consequently, Theorem 1 immediately follows by Corollaries 1 and 2.

\section{Two Examples}

Example 1. For $1 \leq p<2$, we consider the boundary value problem $\left(D_{p}^{\lambda, f}\right)$ with

$$
f(k, t)=p|t|^{p-1} \operatorname{sign}(t)\left(\frac{T+1}{T}+\sin \left(\frac{1}{2 T} \ln \left(|t|^{p}+1\right)\right)+\frac{1}{2 T} \cos \left(\frac{1}{2 T} \ln \left(|t|^{p}+1\right)\right)\right),
$$

for $k \in \mathbb{Z}(1, T)$, then

$$
F(k, t)=\int_{0}^{t} f(k, \tau) d \tau=\frac{T+1}{T}|t|^{p}+\left(|t|^{p}+1\right) \sin \left(\frac{1}{2 T} \ln \left(|t|^{p}+1\right)\right), \text { for } t \in \mathbb{R} .
$$

Since $f(k, t) \geq p t^{p-1}\left(\frac{T+1}{T}-1-\frac{1}{2 T}\right)=\frac{p}{2 T} t^{p-1}>0$, for $t>0$ and $f(k, 0)=0$, we follow that for each fixed $k \in \mathbb{Z}(1, T), F(k, t)$ is strictly monotone increasing on $[0,+\infty)$. One has $\max _{0 \leq s \leq t} F(k, s)=F(k, t)$, for each $t \geq 0$. Clearly,

$$
A_{+\infty}=\liminf _{t \rightarrow+\infty} \frac{T F(k, t)}{t^{p}}=\liminf _{t \rightarrow+\infty} \frac{(T+1) t^{p}+T\left(t^{p}+1\right) \sin \left(\frac{1}{2 T} \ln \left(t^{p}+1\right)\right)}{t^{p}}=1,
$$

and

$$
B^{+\infty}=\limsup _{t \rightarrow+\infty} \frac{T F(k, t)}{t^{p}}=\limsup _{t \rightarrow+\infty} \frac{(T+1) t^{p}+T\left(t^{p}+1\right) \sin \left(\frac{1}{2 T} \ln \left(t^{p}+1\right)\right)}{t^{p}}=2 T+1
$$

In view of $1 \leq p<2$, we follow that $A_{+} \infty<\frac{2^{p-1}}{(T+1)^{\frac{p}{2}}} B^{+\infty}$. Applying to Theorem 2 , problem $\left(D_{p}^{\lambda, f}\right)$ admits an unbounded sequence of positive solutions.

Let us consider another example.

Example 2. Let $T=4, p=3$ andf be a function defined as follows

$$
f(k, t)=3|t| t\left(\frac{5}{4}+\sin \left(\frac{1}{8} \ln \left(|t|^{3}+1\right)\right)+\frac{1}{8} \cos \left(\frac{1}{8} \ln \left(|t|^{3}+1\right)\right)\right), k \in \mathbb{Z}(1,4)
$$


Then, for every $\lambda \in\left(\frac{2 \sqrt{2}}{27}, \frac{8}{75}\right)$, the problem

$$
\left\{\begin{array}{l}
-\triangle\left(\phi_{3, c}(\triangle u(k-1))\right)=\lambda f(k, u(k)), \quad k \in \mathbb{Z}(1,4) \\
u(0)=u(5)=0
\end{array}\right.
$$

Admits an unbounded sequence of positive solutions and an unbounded sequence of negative solutions. Indeed, $f(k, t) \geq 3 t^{2}\left(\frac{5}{4}-1-\frac{1}{8}=\frac{3}{8} t^{2}\right)>0$, for $t>0$ and $f(k, 0)=0$.

$$
F(k, t)=\int_{0}^{t} f(k, \tau) d \tau=\frac{5}{4}|t|^{3}+\left(|t|^{3}+1\right) \sin \left(\frac{1}{8} \ln \left(|t|^{3}+1\right)\right), \text { for } t \in \mathbb{R} .
$$

Since $f(k, t) \geq 3 t^{2}\left(\frac{5}{4}-1-\frac{1}{8}\right)=\frac{3}{8} t^{2}>0$, for $t>0$, we follow that for each fixed $k \in \mathbb{Z}(1,4), F(k, t)$ is strictly monotone increasing on $[0,+\infty)$. Thus, $\max F(k, s)=F(k, t)$, for each $t \geq 0$. Obviously, $|s| \leq t$

$$
A_{ \pm \infty}=\liminf _{t \rightarrow+\infty} \frac{4 F(k, t)}{t^{3}}=\liminf _{t \rightarrow+\infty} \frac{5 t^{3}+4\left(t^{3}+1\right) \sin \left(\frac{1}{8} \ln \left(t^{3}+1\right)\right)}{t^{3}}=1,
$$

and

$$
B^{ \pm \infty}=\limsup _{t \rightarrow+\infty} \frac{4 F(k, t)}{t^{3}}=\limsup _{t \rightarrow+\infty} \frac{5 t^{3}+4\left(t^{3}+1\right) \sin \left(\frac{1}{8} \ln \left(t^{3}+1\right)\right)}{t^{3}}=9 .
$$

Through simple computation, $\max \left\{A_{+\infty}, A_{-\infty}\right\}<\frac{(\sqrt{2})^{p}}{(T+1)^{p-1}} \min \left\{B^{+\infty}, B^{-\infty}\right\}$ holds. Corollary 2 ensures our claim.

\section{Conclusions}

In this paper, we have discussed the Dirichlet boundary value problem of the difference equation with $p$-mean curvature operator. Some sufficient conditions are derived for the existence of sequences of constant-sign solutions to the problem. Two examples are given to show the effectiveness of our results.

To solve problem $\left(D_{p}^{\lambda, f}\right)$, we further develop the methods adopted in [23]. The approaches can be used for the boundary value problems of differential equations involving $p$-mean curvature operator. Therefore, our work has both theoretical and practical significance.

Author Contributions: Conceptualization, J.W.; Formal analysis, J.W. and Z.Z.; Funding acquisition, Z.Z.; Investigation, Z.Z.; Methodology, J.W.; Supervision, Z.Z.; Writing-original draft, J.W.; Writing-review and editing, Z.Z. All authors have read and agreed to the published version of the manuscript.

Funding: This work is supported by the National Natural Science Foundation of China (Grant No. 11971126) and the Program for Changjiang Scholars and Innovative Research Team in University (Grant No. IRT_16R16).

Acknowledgments: The authors wish to thank three anonymous reviewers for their valuable comments and suggestions.

Conflicts of Interest: The authors declare that they have no conflicts of interest.

\section{References}

1. Chen, Z.H.; Shen, Y.T. Infinitely many solutions of Dirichlet problem for $p$-mean curvature operator. Appl. Math. J. Chin. Univ. Ser. B 2003, 18, 161-172. [CrossRef]

2. Napoli, P.D.; Mariani, M.C. Mountain pass solutions to equations of $p$-Laplacian type. Nonlinear Anal. 2003, 54, 1205-1209.

3. Bergner, M. On the Dirichlet problem for the prescribed mean curvature equation over general domains. Differ. Geom. Appl. 2009, 27, 335-343. [CrossRef]

4. Finn, R. Equilibrium Capillary Surfaces; Springer: New York, NY, USA, 1986.

5. Finn, R. On the Equations of Capillarity. J. Math. Fluid Mech. 2001, 3, 139-151. [CrossRef] 
6. Afrouzi, G.A.; Hadjian, A.; Bisci, G.M. A variational approach for one-dimensional prescribed mean curvature problems. J. Aust. Math. Soc. 2014, 97, 145-161. [CrossRef]

7. Bereanu, C.; Mawhin, J. Boundary value problems for second-order nonlinear difference equations with discrete $\phi$-Laplacian and singular $\phi$. J. Differ. Equ. 2008, 14, 1099-1118. [CrossRef]

8. Bonheure, D.; Habets, P.; Obersnel, F.; Omari, P. Classical and non-classical solutions of a prescribed curvature equation. J. Differ. Equ. 2007, 243, 208-237. [CrossRef]

9. Cabada, A.; Otero-Espinar, V. Existence and comparison results for difference $\phi$-Laplacian boundary value problems with lower and upper solutions in reversed order. J. Math. Anal. Appl. 2002, 267, 501-521. [CrossRef]

10. Mawhin, J. Periodic solutions of second order nonlinear difference systems with $\phi$-Laplacian: A variational approach. Nonlinear Anal. 2012, 75, 4672-4687. [CrossRef]

11. Obersnel, F.; Omari, P. Positive solutions of the Dirichlet problem for the prescribed mean curvature equation. J. Differ. Equ. 2010, 249, 1674-1725. [CrossRef]

12. Tolksdorf, P. On the Dirichlet problem for quasilinear equations in domains with conical boundary points. Commun. Partial Differ. Equ. 1983, 8, 773-817. [CrossRef]

13. Agarwal, R.P. Difference Equations and Inequalities, Theory, Methods, and Applications; Marcel Dekker Incorporated: New York, NY, UYA; Basel, Switzerland, 2000.

14. Agarwal, R.P.; Wong, P.J.Y. Advanced Topics in Difference Equations; Kluwer Academic Publishers: Dordrecht, The Netherlands, 1997.

15. Elaydi, S. An Introduction to Difference Equations, 3rd ed.; Springer Verlag: New York, NY, USA, 2011.

16. Kelly, W.G.; Peterson, A.C. Difference Equations: An Introduction with Applications; Academic Press: San Diego, CA, USA; New York, NY, USA; Basel, Switzerland, 1991.

17. Yu, J.S.; Zheng, B. Modeling Wolbachia infection in mosquito population via discrete dynamical model. J. Differ. Equ. Appl. 2019. [CrossRef]

18. Guo, Z.M.; Yu, J.S. The existence of periodic and subharmonic solutions for second-order superlinear difference equations. Sci. China Ser. A Math. 2003, 46, 506-515. [CrossRef]

19. D'Agui, G.; Mawhin, J.; Sciammetta, A. Positive solutions for a discrete two point nonlinear boundary value problem with $p$-Laplacian. J. Math. Anal. Appl. 2017, 447, 383-397.

20. Erbe, L.; Jia, B.G.; Zhang, Q.Q. Homoclinic solutions of discrete nonlinear systems via variational method. J. Appl. Anal. Comput. 2019, 9, 271-294.

21. Kuang, J.H.; Guo, Z.M. Heteroclinic solutions for a class of $p$-Laplacian difference equations with a parameter. Appl. Math. Lett. 2020, 100, 106034. [CrossRef]

22. Lin, G.H.; Zhou, Z.; Yu, J.S. Ground state solutions of discrete asymptotically linear Schrödinger equations with bounded and non-periodic potentials. J. Dyn. Differ. Equ. 2019. [CrossRef]

23. Lin, G.H.; Zhou, Z. Homoclinic solutions of discrete $\phi$-Laplacian equations with mixed nonlinearities. Commun. Pure Appl. Anal. 2018, 17, 1723-1747. [CrossRef]

24. Long, Y.H.; Chen, J.L. Existence of mutiple solutions to second-order discrete Neumann boundary value problems. Appl. Math. Lett. 2018, 83, 7-14. [CrossRef]

25. Long, Y.H.; Wang, S.H. Multiple solutions for nonlinear functional difference equaions by the invariants sets of descending flow. J. Differ. Equ. Appl. 2019, 25, 1768-1789. [CrossRef]

26. Shi, H.P. Periodic and subharmonic solutions for second-order nonlinear difference equations. J. Appl. Math. Comput. 2015, 48, 157-171. [CrossRef]

27. Tang, X.H. Non-Nehari manifold method for periodic discrete superlinear Schrödinger equation. Acta Math. Sin. Engl. Ser. 2016, 32, 463-473. [CrossRef]

28. Zhang, Q.Q. Homoclinic orbits for a class of discrete periodic Hamiltonian systems. Proc. Am. Math. Soc. 2015, 143, 3155-3163. [CrossRef]

29. Zhang, Q.Q. Homoclinic orbits for discrete Hamiltonian systems with indefinite linear part. Commun. Pure Appl. Anal. 2017, 14, 1929-1940. [CrossRef]

30. Zhang, Q.Q. Homoclinic orbits for discrete Hamiltonian systems with local super-quadratic conditions. Commun. Pure Appl. Anal. 2019, 18, 425-434. [CrossRef]

31. Zhou, Z.; Ma, D.F. Multiplicity results of breathers for the discrete nonlinear Schrödinger equations with unbounded potentials. Sci. China Math. 2015, 58, 781-790. [CrossRef] 
32. Zhou, Z.; Su, M.T. Boundary value problems for $2 n$-order $\phi_{c}$-Laplacian difference equations containing both advance and retardation. Appl. Math. Lett. 2015, 41,7-11. [CrossRef]

33. Zhou, Z.; Yu, J.S. Homoclinic solutions in periodic nonlinear difference equations with superlinear nonlinearity. Acta Math. Sin. Engl. Ser. 2013, 29, 1809-1822. [CrossRef]

34. Zhou, Z.; Yu, J.S.; Chen, Y.M. Homoclinic solutions in periodic difference equations with saturable nonlinearity. Sci. China Math. 2011, 54, 83-93. [CrossRef]

35. Zhou, Z.; Ling, J.X. Infinitely many positive solutions for a discrete two point nonlinear boundary value problem with $\phi_{c}$-Laplacian. Appl. Math. Lett. 2019, 91, 28-34. [CrossRef]

36. Bonanno, G. A critical point theorem via the Ekeland variational principle. Nonlinear Anal. 2012, 75, $2992-3007$. [CrossRef]

37. Bonanno, G.; Candito, P. Infinitely many solutions for a class of discrete non-linear boundary value problems. Appl. Anal. 2009, 88, 605-616. [CrossRef]

38. Bonanno, G.; Candito, P.; D'Agui, G. Variational methods on finite dimensional Banach spaces and discrete problems. Adv. Nonlinear Stud. 2014, 14, 915-939. [CrossRef]

39. Bonanno, G.; Jebelean, P.; Serban, C. Superlinear discrete problems. Appl. Math. Lett. 2016, 52, $162-168$. [CrossRef]

40. Marano, S.A.; Motreanu, D. Infinitely many critical points of non-differentiable functions and applications to a Neumann type problem involving the p-Laplacian. J. Differ. Equ. 2002, 182, 108-120. [CrossRef]

41. Hardy, G.H.; Littewood, J.E.; Pólya, G. Inequalities, 2nd ed.; Cambridge University Press: Cambridge, UK, 1988.

42. Jiang, L.Q.; Zhou, Z. Three solutions to Dirichlet boundary value problems for $p$-Laplacian difference equations. Adv. Differ. Equ. 2008, 2008, 1-10. [CrossRef]

(C) 2020 by the authors. Licensee MDPI, Basel, Switzerland. This article is an open access article distributed under the terms and conditions of the Creative Commons Attribution (CC BY) license (http://creativecommons.org/licenses/by/4.0/). 